\title{
Multitone Interference of Fast FH/MFSK Systems Over Ricean Fading Channels
}

\author{
Chen Jiang and Jiangzhou Wang, Senior Member, IEEE
}

\begin{abstract}
The effects of channel fading of multitone interference on the performance of multiple hops per symbol fast frequencyhopped (FFH), $M$-ary orthogonal frequency-shift keyed (MFSK) noncoherent systems are analytically investigated. The multiple equal-power interference tones are assumed to correspond to some of the possible FFH/M-ary orthogonal signaling tones. It is also assumed that channel fading characteristics are independent for the signal tone and interference tone. We evaluate how the effect of the multitone interference fading on system performance is changed by various parameters, such as the number of interference tones, the number of hops per symbol and the modulation order. In addition, our numerical results indicate that the larger number of hops per symbol makes the system performance more sensitive to the fading of multitone interference.
\end{abstract}

\section{INTRODUCTION}

Frequency hopping $(\mathrm{FH})$ has been widely studied for various applications [1-7]. In FH systems, interference and jamming models mainly include thermal wideband noise, multitone jamming, partial-band jamming and multiple access interference. Robertson et. al. [1] and Levitt [2] analyzed the performance degradation resulting from multitone interference of FH/MFSK for Ricean fading channel, where one or more symbols per hop are transmitted. References [3,4] overviewed the error probability performance of noncoherent frequencyshift-keyed $M$-ary systems under tone interference, where the channel fading is neglected. The effectiveness of partial-band noise jamming as an electronic countermeasure against fast frequency-hopped binary or $M$-ary frequency shift keyed (BFSK or MFSK) has been widely documented in which, [5] is for no-fading channels and [6] is for Ricean fading channels.

In the previous work, the effect of multitone interference on the performance of noncoherent FH/FSK has limited to the one symbol per hop. This paper investigates the performance degradation resulting of orthogonal noncoherent fast frequency hopping MFSK with multitone interference. The multiple equalpower interference tones are assumed to correspond to some of the possible FFH/MFSK orthogonal signaling tones. Furthermore, the channel for each hop band is modeled as an independent, frequency-nonselective, slowly fading Ricean process. It is also assumed that channel fading characteristics are independent for the signal tone and interference tone.

\section{A. FFH/MFSK system model}

\section{MODEL}

We consider a communication system whose fundamental requirement is to transmit the source information of binary data sequence by means of $M$-ary frequency shift-keying (MFSK) over the channel, where one $M$-ary symbol is represented by one of $M=2^{k}$ orthogonal tone, $M$ is order of MFSK modulation and $k$ represents the number of bits per symbol transmitted. It is assumed that the input binary data have a period of $T_{b}$ and the symbol duration is $T_{s}=k T_{b}$. Finally, the symbols are mixed with frequency-hopping tone of frequency $f_{h}$ and duration $T_{h}$. In FFH/MFSK system, an $L$ hops per symbol scheme is considered, in which one symbol is broken into $L$ independent transmissions of duration $T_{S} / L$. So the input and output periods are related by $T_{k}=k T_{b} / L$. The signal transmitted is given by

$S_{m}(t)=\operatorname{Re}\left\{\sqrt{2 P} \exp \left[j\left\{2 \pi\left[f_{h_{i}}+(m-1) \Delta f\right] t+\theta_{m}\right\}\right]\right\}$,

where $m=1,2, \ldots, M . \operatorname{Re}\{$.$\} stands for real part and j=\sqrt{-1}$. $P$ is transmitted signal power. $\Delta f$ is chosen as the hop rate $R_{h}=1 / T_{h}$ in order that these tones are orthogonal, $i=1,2$, $\ldots, N$ designates the possible discrete frequency available for hopping and $\theta_{m}$ is unknown phase.

The receiver (Fig.1) consists of a frequency dehopper, a bank of $M$ matched filters and quadratic detectors. The output of each filter/detector is sampled every $T_{h}$ and a decision is made as to which of the $M$ samples is largest. The diversity combining is done before the decision device.

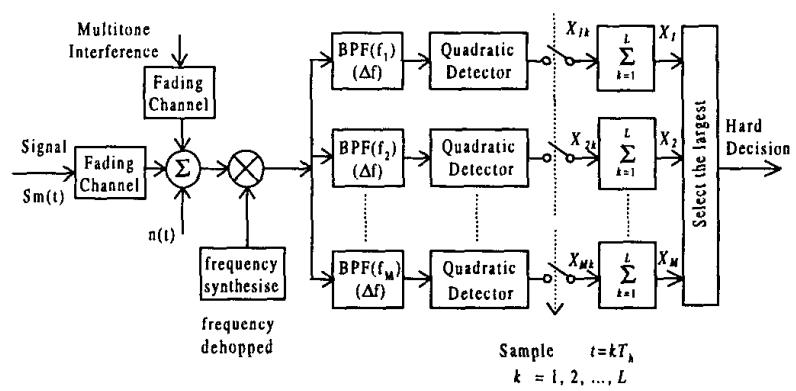

Fig.1 A detailed model of the frequency-dehopper and the MFSK demodulator.

\section{B. Channel model}

The total bandwidth $W_{T}$ of the FFH/MFSK system can be divided into $N$ FH bands ( $W_{\text {band }}$ ) and each FH band can be further divided into $M$ bins. Therefore, $W_{T}=N \times W_{\text {band }}=M \times N$ $\times \Delta f$. It is assumed that the signal during each hop of one symbol fades independently [7]. In addition, the channel for each hop of a symbol is modeled as a slowly fading Ricean process. As a result, the signal amplitude can be modeled as a Ricean random variable that remains constant at least for the duration of a single hop.

Both the signal tones and the multiple interference tones are 
assumed to be affected by channel fading. It is also assumed that channel fading characteristics are independent for the signal tone and interference tone.

\section{Interference model}

It is assumed that the channel is jammed by the multitone interference which has a total jamming power $P_{J T}$ and consists of $q$ equal power interference tones spread randomly over system bandwidth $W_{T}$. Hence, the power of a single interference tone is $P_{J}=P_{J t} / q$. It is also assumed that the multiple interference tones are transmitted at frequencies exactly corresponding to the various $M \times N$ FFH/MFSK signal tones and only one of $M$ channels of receiver is possible jammed by an interference tone during one hopping. In fact, [8] has shown that such case of multitone distribution is related to Houston's worst-case multitone jamming.

The thermal noise is modeled as AWGN, and its power spectral density is defined as $N_{0} / 2$.

\section{ANALYSIS}

To obtain the probability of symbol error $P_{S E}$, we need to average the conditional probability of symbol error over all of possible multitone jamming pattern combinations. $l_{0}$ is defined as the number of hops, over which all of $\dot{M}$ channels of the receiver are not jammed by interference tone during the observed symbol interval, $l_{s}$ is defined as the number of hops, over which the channel containing the signal is jammed. In addition, for the other $M-1$ channels without the signal, $m_{0}$ is defined as the number of channels, which are not jammed over all $L$ hops and $m_{1}, m_{2}, \ldots, m_{L}$ are defined as the number of channels, which have one hop, two hops,..., $L$ hops, respectively, to be jammed. Suppose each hop is jammed at most by one interference tone. Hence,

$$
l_{0}+l_{s}+\sum_{l=1}^{L}\left(m_{l} \times l\right)=L
$$

and

$$
\sum_{l=0}^{L} m_{l}=M-1,
$$

where all of $l_{0}, l_{S}$, and $m_{l}$ are positive integers. It is assumed the vector

$$
K=\left(l_{0}, l_{s}, m_{0}, m_{1}, \ldots m_{L}\right)
$$

satisfies the relation (2a) and (2b). Therefore, any possible jamming pattern in the $M$ channels over $L$ hops can be described by a corresponding element of vector $K$. Since vector $K$ has a variety of combinations which will lead to a different conditional probability of error, we need to average the conditional probability of symbol error over all the possible combinations to obtain the probability of symbol error, which is given by

$$
P_{S E}=\sum_{K} P_{r}(K) \times\left. P_{S E}\right|_{K}
$$

where $P_{r}(K)$ is the probability of the jamming pattern described by the vector $K$, which satisfies (2a) and (2b), and $\left.P_{S E}\right|_{K}$ is the conditional probability of symbol error, conditioned or the jamming pattern described by the vector $K . P_{r}(K)$ and $\left.P_{S E}\right|_{K}$ will be described in more detail.

\section{A. The probability of the jamming pattern described by the vector $K$}

Since the FFH/MFSK receiver observes the output of the $M$ receiver channels over $L$ hops for the symbol decision process, all possible two dimensional assignment of the janming patterns in the $M$ channels over $L$ hops should be considered. Note that there are $\left(\begin{array}{c}M-1 \\ m_{0}, m_{1}, \ldots m_{L}\end{array}\right)$ ways to form vector $K$. over the $M$ channel and $(l_{0}, l_{s}, \underbrace{1, \ldots}_{m_{1}}, \underbrace{2, \ldots 2}_{m_{2}}, \ldots \underbrace{L, \ldots L}_{m_{L}})$ ways to form vector $K$ over the $L$ hops. In addition, the probability that all of $M$ channels are not jammed by interference tone during one hop is $1-q / N$, and the probability that the specific one of $M$ channels during one hop is jammed is $q /(N M)$. Therefore, $P_{r}(K)$ is given by

$$
\begin{aligned}
P_{r}(K)= & (l_{0}, l_{s}, \underbrace{1, \ldots, 1}_{m_{1}}, \underbrace{2, \ldots 2}_{m_{2}}, \ldots \underbrace{L, \ldots L L}_{m_{L}}) \cdot\left(\begin{array}{c}
M-1 \\
m_{0}, m_{1}, \ldots m_{L}
\end{array}\right) \\
& \cdot\left(1-\frac{q}{N}\right)^{l_{0}} \cdot\left(\frac{q}{M N}\right)^{L-l_{0}} \\
= & \frac{L !}{l_{0} ! \cdot l_{s} ! \cdot \prod_{l=1}^{L}(l !)^{m_{l}}} \cdot \frac{(M-1) !}{\prod_{l=0}^{L} m_{l} !} \cdot\left(1-\frac{q}{N}\right)^{l_{0}} \cdot\left(\frac{q}{M N}\right)^{L-l_{0}} .
\end{aligned}
$$

B. The conditional probability of symbol error, conditicned on the jamming pattern described by the vector $K$

As shown in Fig.1, $\left\{X_{m} ; 1 \leq m \leq M\right\}$ is the set of decision random variables, where $m$ means the $m$ th receiver chanrel. The conditional probability of symbol error, conditioned on the jamming pattern described by the vector $K$, can be obtained by assuming that the useful signal is present in channel 1 of the receiver and the channel $m, 2+\sum_{i=0}^{l} m_{i}-m_{l} \leq m<2+\sum_{i=0}^{l} m_{i}$, is jammed by the interference tone over $l$ out of $L$ hops during the observed symbol interval. Therefore, $\left.X_{m}\right|_{K}$ can be rewritten as

$$
X_{1} l_{K}=X_{1} l_{s}
$$

and

$$
\left.X_{m}\right|_{K}=X_{m} l_{l}, \quad 2+\sum_{i=0}^{l} m_{i}-m_{l} \leq m<2+\sum_{i=0}^{l} m_{i} .
$$

Note that $X_{m}, m \neq 1$, does not contain the useful signal. Therefore, the conditional probability of symbol error, conditioned on the jamming pattern described by the vector $K$ is 
given by

$$
\begin{aligned}
P_{S E \mid K} & =P_{r}\left[\bigcup_{m=2}^{M}\left(\left.X_{1}\right|_{K}<\left.X_{m}\right|_{K}\right)\right] \\
& =1-P_{r}\left\{\bigcap_{l=0}^{L}\left[\bigcap_{m=2+\sum_{i=0}^{1} m_{i}-m_{l}}^{1+\sum_{i=0}^{1} m_{i}}\left(\left.X_{1}\right|_{l_{s}}>\left.X_{m}\right|_{l}\right)\right]\right\} .
\end{aligned}
$$

It is assumed that $f_{X_{m}}\left(x_{m}\right)$ is the probability density function of the random variable $X_{m}$. Since the outputs of each channel of the FFH/MFSK receiver are assumed to be independent and since each $X_{m} l_{l}, 2+\sum_{i=0}^{l} m_{i}-m_{l} \leq m<2+\sum_{i=0}^{l} m_{i}$, is assumed to be identical, (6) can be rewritten as

$$
\begin{aligned}
\left.P_{S E}\right|_{K} & =1-\int_{0}^{\infty} f_{X_{1}}\left(x_{1} \mid l_{S}\right) \prod_{l=0}^{L}\left[\int_{0}^{x_{1}} f_{X_{m}}\left(x_{m} \mid l\right) d x_{m}\right]^{m_{l}} d x_{1} \\
& =1-\int_{0}^{\infty} f_{X_{1}}\left(x_{1} \mid l_{S}\right) \prod_{l=0}^{L}\left[1-z_{l}\right]^{m_{l}} d x_{1},
\end{aligned}
$$

where $z_{l}$ is defined as

$$
z_{l}=\int_{x_{1}}^{\infty} f_{X_{m}}\left(x_{m} \mid l\right) d x_{m} .
$$

Since most often, $0<z_{l} \ll 1,\left.\quad P_{S E}\right|_{K}$ can be simplified, using the following inequality:

$$
\prod_{l=0}^{L}\left(1-z_{l}\right)^{m_{l}} \geq \prod_{l=0}^{L}\left(1-m_{l} z_{l}\right) \geq 1-\sum_{l=0}^{L} m_{l} z_{l} .
$$

Therefore, the upper bound of $\left.P_{S E}\right|_{K}$ is given by

$$
\left.P_{S E}\right|_{K} \leq \sum_{l=0}^{L}\left[m_{l} \int_{0}^{\infty} \int_{0}^{\infty} f_{X_{1}}\left(x_{1} \mid l_{S}\right) f_{X_{m}}\left(x_{1}+x_{m} \mid l\right) d x_{1} d x_{m}\right] \text {. }
$$

\section{Probability density functions $f_{X_{1}}\left(x_{1} \mid l_{S}\right)$ and $f_{X_{m}}\left(x_{m} \mid l\right)$}

As shown from Fig.1, the random variable $X_{m}$ that represents the output of the channel after diversity combining is given as

$$
X_{m}=\sum_{k=1}^{L} X_{m k}
$$

where $X_{m k}, 1 \leq k \leq L$, is the $m$ th channel output of the receiver for hop $k$ of a symbol. This channel either contains the signal or not and either is jammed or not by interference tone during the hop. The former case will be considered first. When the channel containing the signal is jammed by a interference tone during the hop $k$, it will be assumed that the frequencies of the signal tone and interference tone are identical and their phase difference is a random variable, uniformly distributed in $[0,2 \pi]$. Thus, the sum of the signal tone and interference tone can be expressed as a single composite sine wave. The power of the composite signal at the channel output has the value

$$
a^{2}=a_{S}^{2}+a_{J}^{2}+2 a_{S} a_{J} \cos \theta,
$$

where $a_{S}^{2}$ and $a_{j}^{2}$ are the powers of the signal and interference tone, respectively, at the output of the detector. $\theta$ is the phase difference between the signal tone and interference tone. Since the random variable $X_{1 k}$ is the sum of composite signal and the receiver background thermal noise at the channel output for hop $k$ of a symbol, $X_{1 k}$ has the probability density function of the square of the envelope of a sine wave with random phase plus a narrowband Gaussian process. The result is a special case of the non-central chi-squared distribution. For a value of composite signal amplitude $\sqrt{2} a$, the pdf is given by

$f_{X_{1 k}}\left(x_{1 k} \mid\right.$ hop jammed,$\left.a\right)=\frac{1}{2 \sigma_{N}^{2}} \exp \left(-\frac{x_{1 k}+2 a^{2}}{2 \sigma_{N}^{2}}\right) I_{0}\left(\frac{a \sqrt{2 x_{1 k}}}{\sigma_{N}^{2}}\right)$,

$x_{1 k \geq 0}$,

where $\sigma_{N}^{2}=N_{0} \Delta f=N_{0} R_{h}$ is the thermal noise power. The conditional characteristic function corresponding to this pdf is $\psi_{X_{1 k}}(j v \mid$ hop jammed, $a)=$

$$
\frac{1}{1-j 2 \sigma_{N}^{2} \nu} \exp \left(\frac{j 2\left(a_{S}^{2}+a_{J}^{2}+2 a_{S} a_{J} \cos \theta\right) v}{1-j 2 \sigma_{N}^{2} \nu}\right) \text {. }
$$

In order to obtain the characteristic function for $X_{1 k}$ should be averaged over $a$, given by (12). Thus,

$$
\begin{array}{r}
\psi_{X_{1 k}}(j v \mid \text { hop jammed })=\frac{1}{2 \pi} \int_{0}^{2 \pi} \int_{0}^{\infty} f_{A_{S}}\left(a_{S}\right) \int_{0}^{\infty} f_{A_{J}}\left(a_{J}\right) \\
\cdot \psi_{X_{1 k}}(j v \mid \text { hop jammed, } a) d a_{J} d a_{S} d \theta .
\end{array}
$$

Since the Ricean fading is assumed for both signal and multitone interference, the amplitude pdf's of the signal tone and the interference tones are Ricean pdf, given by

$$
\begin{array}{ll}
f_{A_{S}}\left(a_{s}\right)=\frac{a_{S}}{\sigma_{S}^{2}} \exp \left(-\frac{a_{S}^{2}+\alpha_{S}^{2}}{2 \sigma_{S}^{2}}\right) I_{0}\left(\frac{a_{S} \alpha_{S}}{\sigma_{S}^{2}}\right), & a_{S} \geq 0, \\
f_{A_{J}}\left(a_{J}\right)=\frac{a_{J}}{\sigma_{J}^{2}} \exp \left(-\frac{a_{J}^{2}+\alpha_{J}^{2}}{2 \sigma_{J}^{2}}\right) I_{0}\left(\frac{a_{J} \alpha_{J}}{\sigma_{J}^{2}}\right), & a_{J} \geq 0,
\end{array}
$$

where $\alpha_{S}^{2}$ and $2 \sigma_{S}^{2}$ are the powers of the direct and diffuse component of the signal, respectively. $\alpha_{J}^{2}$ and $2 \sigma_{J}^{2}$ are the powers of the direct and diffuse component of the interference tone, respectively. The total average power of signal and one interference are $P_{S}=\alpha_{S}^{2}+2 \sigma_{S}^{2}$ and $P_{J}=P_{J T} / q=\alpha_{J}^{2}+2 \sigma_{J}^{2}$. Substituting (14), (16) and (17) into (15) and utilizing (6.633.4) of [9] , (15) can be simplified by

$$
\begin{aligned}
& \psi_{X_{1 k}}(j v \mid \text { hop jammed })=\frac{1}{1-j 2\left(2 \sigma_{S}^{2}+2 \sigma_{J}^{2}+\sigma_{N}^{2}\right) v} \\
& \cdot \exp \left[\frac{j 2\left(\alpha_{S}^{2}+\alpha_{J}^{2}\right) v}{1-j 2\left(2 \sigma_{S}^{2}+2 \sigma_{J}^{2}+\sigma_{N}^{2}\right) v}\right] J_{0}\left[\frac{4 \alpha_{S} \alpha_{J} v}{1-j 2\left(2 \sigma_{S}^{2}+2 \sigma_{J}^{2}+\sigma_{N}^{2}\right) v}\right] .
\end{aligned}
$$

When the channel containing the signal is not jammed by interference tone during hop $k$, substituting $\alpha_{J}=\sigma_{J}=0$ into (18), one obtains 
$\psi_{X_{1 k}}(j v \mid$ hop not jammed $)=$

$$
\frac{1}{1-j 2\left(2 \sigma_{S}^{2}+\sigma_{N}^{2}\right) \nu} \cdot \exp \left[\frac{j 2 \alpha_{S}^{2} \nu}{1-j 2\left(2 \sigma_{S}^{2}+\sigma_{N}^{2}\right) v}\right]
$$

Since the interference tone and noise are assumed independent from hop to hop, the randcm variables $\psi_{X_{1 k}}$ are independent from hop to hop. Therefore, when the channel containing the signal is jammed over $l_{S}$ hops and is not jammed over $L-l_{S}$ hops during the observed symbol interval, the conditional characteristic function of the random variable $X_{1}$, defined by (11) is

$$
\begin{aligned}
& \psi_{X_{1}}\left(j v \mid l_{S}\right)= \\
& \left(\frac{1}{1-j 2\left(2 \sigma_{S}^{2}+\sigma_{N}^{2}\right) v}\right)^{L-l_{S}}\left(\frac{1}{1-j 2\left(2 \sigma_{S}^{2}+2 \sigma_{J}^{2}+\sigma_{N}^{2}\right) v}\right)^{l_{s}} \\
& \cdot \exp \left(\frac{j 2 \alpha_{S}^{2}\left(L-l_{S}\right) v}{1-j 2\left(2 \sigma_{S}^{2}+\sigma_{N}^{2}\right) v}+\frac{j 2\left(\alpha_{S}^{2}+\alpha_{J}^{2}\right) l_{S} v}{1-j 2\left(2 \sigma_{S}^{2}+2 \sigma_{J}^{2}+\sigma_{N}^{2}\right) v}\right) \\
& {\left[J_{0}\left[\frac{4 \alpha_{S} \alpha_{J}}{1-j 2\left(2 \sigma_{S}^{2}+2 \sigma_{J}^{2}+\sigma_{N}^{2}\right) v}\right]\right]^{l_{S}} .}
\end{aligned}
$$

Similarly, using the above result, the conditional characteristic function of the random variable $X_{m}{ }_{1}, m \neq 1$, can be obtained.

Since $X_{m} l_{l}, m \neq 1$, does not contain the signal and is jammed over $l$ hops during the observed symbol interval, substituting $\alpha_{s}=\sigma_{S}=0$ and $l_{S}$ with $l$ in (20), the conditional characteristic function of the random variable $X_{m} l_{l}$ is given by

$$
\begin{aligned}
\psi_{X_{m}}(j v \mid l) & =\left(\frac{1}{1-j 2 \sigma_{N}^{2} v}\right)^{L-l}\left(\frac{1}{1-j 2\left(2 \sigma_{J}^{2}+\sigma_{N}^{2}\right) v}\right)^{l} \\
& \cdot \exp \left(\frac{j 2 \alpha_{J}^{2} l v}{1-j 2\left(2 \sigma_{J}^{2}+\sigma_{N}^{2}\right) v}\right) .
\end{aligned}
$$

Finally, the conditional probability of symbol error in (10), conditioned on the jamming pattern (vector $K$ ) can be obtained by means of the convolution function

$$
\begin{aligned}
& \int_{0}^{\infty} f_{X_{1}}\left(x_{1} \mid l_{S}\right) f_{X_{m}}\left(x_{1}+x_{m} \mid l\right) d x_{1}= \\
& \frac{1}{2 \pi} \int_{-\infty}^{\infty} \psi_{X_{1}}\left(-j v \mid l_{S}\right) \psi_{X_{m}}(j v l l) \exp \left(-j v x_{m}\right) d v .
\end{aligned}
$$

Substituting (22) into (10), $\left.P_{S E}\right|_{K}$ is given by

$$
\begin{aligned}
\left.P_{S E}\right|_{K}=\frac{1}{2 \pi} \sum_{l=0}^{L} & {\left[m_{l} \int_{0}^{\infty} \int_{-\infty}^{\infty} \psi_{X_{1}}\left(-j v \mid l_{S}\right) \psi_{X_{m}}(j v \mid l)\right.} \\
\cdot & \left.\exp \left(-j v x_{m}\right) d v d x_{m}\right] .
\end{aligned}
$$

By means of the following equation in (17.23) of [9]

$$
\int_{0}^{\infty} \exp (-j v x) d x=\pi \delta(v)+\frac{1}{j v},
$$

where $\delta(v)$ represents the conventional delta function, we have

$$
\left.P_{S E}\right|_{K}=\frac{1}{2 \pi} \sum_{l=0}^{L}\left\{m_{l}\left[\pi+\int_{-\infty}^{\infty} \frac{1}{j v} \psi_{X_{1}}\left(-j v \mid l_{S}\right) \psi_{X_{m}}(j v \mid l) d v\right]\right\} \text {. }
$$

Substituting (25) into (3), $P_{S E}$ is given by

$$
\begin{aligned}
P_{S E} & =\frac{1}{2 \pi} \sum_{K} \sum_{l=0}^{L}\left\{m_{l} P_{r}(K)[\pi\right. \\
& \left.\left.+2 \int_{0^{+}}^{\infty} \operatorname{Re}\left\{\frac{1}{j v} \psi_{X_{1}}\left(-j v \mid l_{S}\right) \psi_{x_{m}}(j \nu \mid l)\right\} d v\right]\right\}
\end{aligned}
$$

where $0^{+}$stands for an extremely small positive value.

Once the symbol error probability $P_{S E}$ has been obtained, the corresponding bit error rate $P_{B E}$ is given by

$$
P_{B E}=\frac{M / 2}{M-1} P_{S E}
$$

\section{NUMERICAL RESULTS AND CONCLUSIONS;}

The effect of the fading of multitone interference on FFH/MFSK system performance is investigated. For a fair comparison, it is assumed that the total FH bands $N=1000$ is fixed.

Firstly, the performance of FFH/MFSK system as a function of the ratio $(q / N)$ of the number of the interference tones to the total FH bands is shown in Fig.2. Where $L=4, M=4, P_{S}$ $/ P_{J T}$ (the signal to the total multitone interference power ratio) $=-25 d B$ and $E_{b} / N_{0}=16 d B$. The signal experiences Ricean

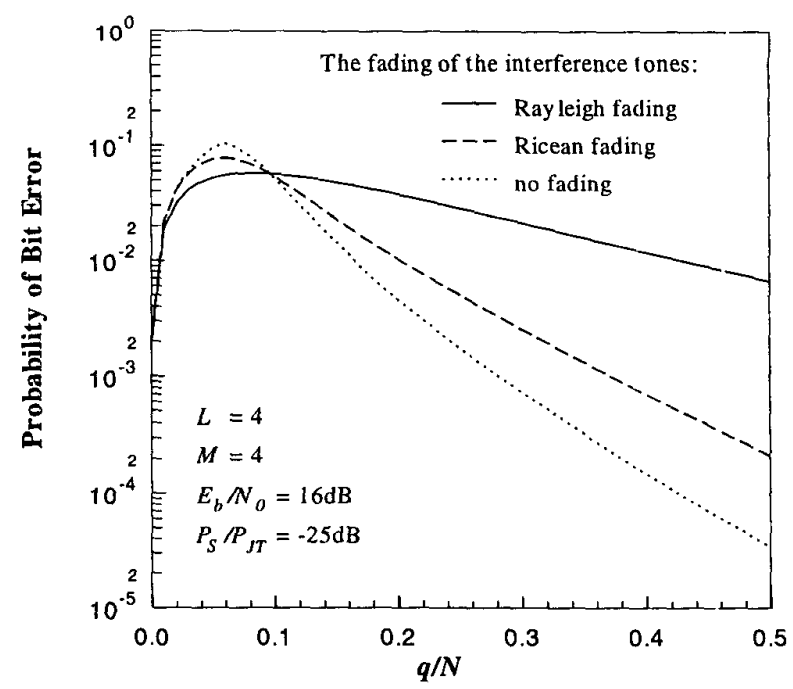

Fig.2 Probability of bit error versus the ratio ot: the number of interference tones to the total $\mathrm{FH}$ bands $(q / N)$ with Ricean fading of the signal. 


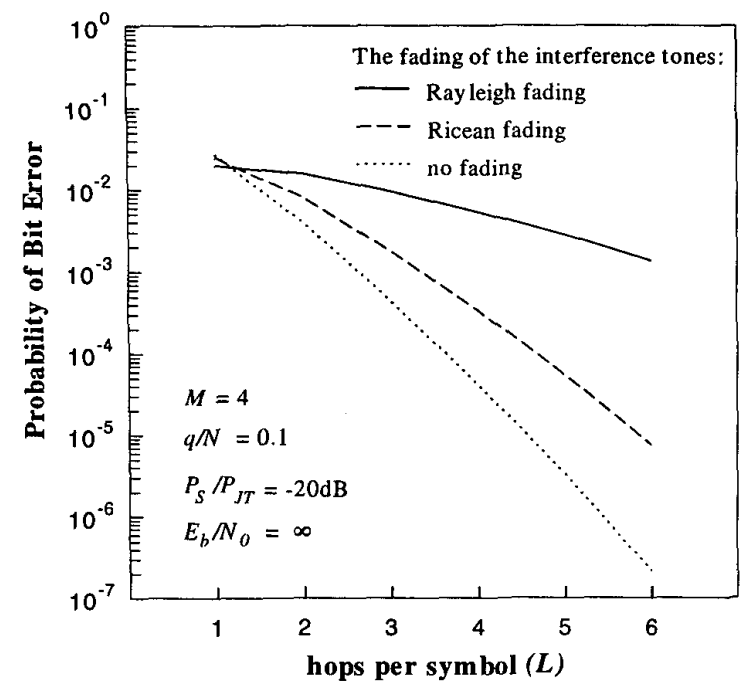

Fig.3 Probability of bit error versus the number of hops/symbol (L) with Ricean fading of the signal.

fading with $\alpha_{S}^{2} / 2 \sigma_{S}^{2}=10 d B$. The multitone interference experiences either no fading with $\alpha_{J}^{2} / 2 \sigma_{J}^{2}=40 d B$, or Ricean fading with $\alpha_{J}^{2} / 2 \sigma_{J}^{2}=10 d B$, or nearly Rayleigh fading with $\alpha_{J}^{2} / 2 \sigma_{J}^{2}=-20 d B$. It is shown from the figure that there is a typical value $\left(q_{w o r s t} / N\right)$ of $q / N$, which maximizes the probability of bit error. For the typical value of the signal to the total multitone power ratio, $q_{\text {worst }} / N$ is about 0.06 . It can also be seen the worst case performance of FFH/MFSK receiver becomes litter advantage when the interference tones experiences Rayleigh fading. However, when $q$ is increased, the performance with no fading of interference tones improves much more than with Rayleigh fading of interference tones.

The effect of interference fading on the system performance as a function of the number of hops per symbol $L$ when the signal experiences Ricean fading $\left(\alpha_{S}^{2} / 2 \sigma_{S}^{2}=10 \mathrm{~dB}\right)$ is illustrated in Fig.3. In order that the effect of interference fading on the system performance is more clearly shown, the thermal and other wideband noise are neglected $\left(E_{b} / N_{o} \rightarrow \infty\right)$. It can be seen that the use of larger number of hops per symbol $(L)$ makes the system performance more sensitive to the fading of multitone interference. In other words, when the interference tones experiences channel fading, the improvement in system performance due to larger value of $L$ is reduced significantly.

Fig.4 shows the effect of the interference fading on the performance of FFH/MFSK system with Ricean fading of the signal ( $a_{s}^{2} / 2 \sigma_{s}^{2}=10 d B$ ) as a function of the modulation order $M$. It is seen that when the interference tones experience Rayleigh fading, increasing $M$ is not helpful for the performance. However, when interference tones do not

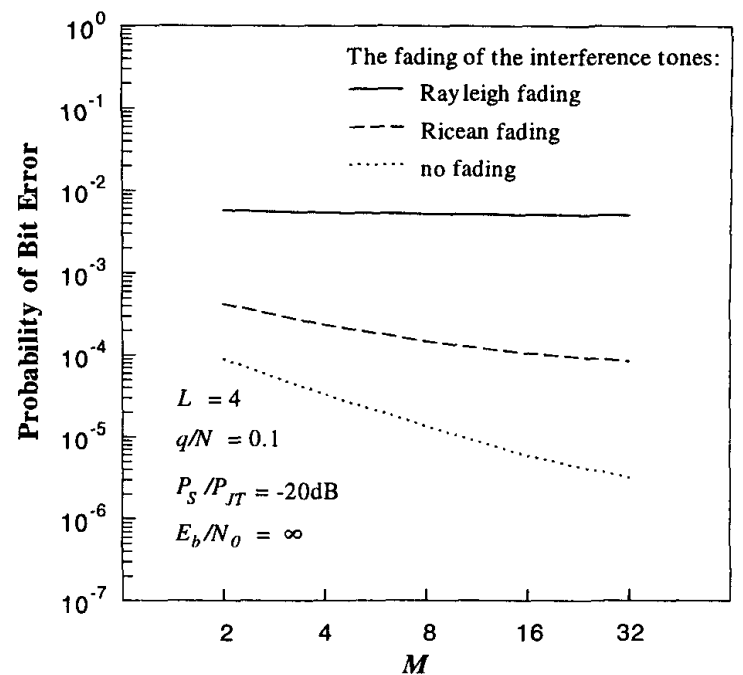

Fig.4 Probability of bit error versus the modulation order (M) with Ricean fading of the signal.

experience fading, increasing $M$ means improves the performance.

Acknowledgments: The authors would like to thank to the Research Grant Council (RGC) of the Hong Kong Government and CRCG of the University of Hong Kong.

\section{REFERENCES}

[1] R. Clark Robertson and Joseph F. Sheltry "Multiple tone interference of frequency-hopped noncoherent MFSK signals transmitted over Ricean fading channels," IEEE Trans. Commun., vol.44, pp.867-875, July 1996.

[2] B. K. Levitt "FH/MFSK performance in Multitone jamming" IEEE $J$. Select. Areas Commun., vol. SAC-3, pp. 627-643, Sept. 1985.

[3] M. J. Massaro, "Error performance of M-ary noncoherent FSK in the presence of CW tone interference," IEEE Trans. Commun., vol. COM-23, pp.1367-1369, Nov. 1975.

[4] J. S. Bird and E. B. Felsted, "Antijam performance of fast frequencyhopped M-ary NCFSK-An overview," IEEE J. Select. Areas Commun. Vol. SAC-4, pp.216-233, Mar. 1986.

[5] J. S. Lee, R. H. French, and L. E. Miller, "Probability of error analyses of a BFSK frequency-hopping system with diversity under partial-band jamming interference-Part I: Performance of square-law linear combining soft decision receiver," IEEE Trans. Commun., vol. COM-32, pp.645-653 June 1984.

[6] C. D. Chung and P. C. Huang "Effect of fading and partial-band noise jamming on a fast FH/BFSK acquisition receiver with noise-normalization combination," IEEE Trans. Commun., vol.44, pp.94-104, Jan. 1996.

[7] C. M. Keller and M. B. Pursley "Diversity Combining for Channels with Fading and Partial-Band Interference," IEEE J. Select. Areas Commun. Vol. SAC-5, pp.248-260, Feb. 1987.

[8] S. W. Houston, "Modulation techniques for communications, Part 1: Tone and noise jamming performance of spread spectrum M-ary FSK and 2,4ary DPSK waveforms," in IEEE NAECON 75 Rec., 1975, pp. 51-58.

[9] I. S. Gradshteyn and I. M. Ryzhik, "Table of Integration, Series and Productions," $5^{\text {nd }}$ ed. CD-Rom version 1.0, Academic Press, 1996. 